\title{
De novo T-cell generation in patients at different ages and stages of HIV-1 disease
}

Massimo Nobile, Rafael Correa, José A. M. Borghans, Claudia D’Agostino, Philippe Schneider, Rob J. de Boer, and Giuseppe Pantaleo, for the Swiss HIV Cohort Study

\begin{abstract}
We assessed de novo T-cell generation by determining T-cell receptor-rearrangement excision circles (TRECs) based on patient age and on stage of HIV-1 infection. TRECs were measured in purified CD4 and CD8 T cells of a large cohort of HIV-1-infected subjects $(n=297)$ with chronic infection but no previous antiretroviral treatment and of a control group of HIV-negative subjects $(n=120)$. HIV-1infected subjects were stratified on the basis of CD4 T-cell counts in 3 groups, early-stage disease (more than 500 CD4 T
\end{abstract}

cells), intermediate-stage disease (200500 CD4 T cells), and late-stage disease (fewer than 200 CD4 T cells). Compared with the control group, CD8 TREC contents were severely reduced $(P<.001)$ in HIV-1-infected subjects regardless of the stage of HIV disease. In contrast, CD4 TREC contents were significantly increased $(P=.003)$ in HIV-1-infected subjects during early-stage disease, similar at intermediate-stage disease, and severely reduced only at late-stage disease. We show that the increase in CD4 TRECs was mostly limited to younger (younger than $\mathbf{4 5}$ years) patients at early-stage disease. Our results demonstrate a dichotomy between TREC contents in CD4 and CD8 T-cell populations in HIV-1 infection and indicate that thymus function in younger subjects is preserved at early and intermediate stages of HIV infection. (Blood. 2004;104:470-477)

(c) 2004 by The American Society of Hematology

\section{Introduction}

Major advances have recently been made in the study of thymopoiesis and generation of naive peripheral $\mathrm{T}$ cells by the evaluation of recent thymic emigrants (RTEs) through the development of an assay that quantifies T-cell receptor excision circles (TRECs). ${ }^{1}$ Most previous studies measured TRECs produced during the rearrangement of the $\alpha$-chain of the T-cell receptor (TCR), the so-called $\mathrm{V} \alpha$ signal joint (Sj) TRECs. These DNA circles are not replicated during cell mitosis and are, therefore, diluted by cell division. Because TREC frequencies can be influenced by a series of factors, including the extent of cell proliferation and the longevity of naive $\mathrm{T}$ cells, interpreting TREC data to evaluate thymic function must be done with great care. ${ }^{2}$

TREC contents have been extensively measured in HIV-1 infection. In 1998, Douek et $\mathrm{al}^{3}$ provided evidence for the existence of a functional thymus in adults and suggested reduced thymic output in HIV-1 infection. TREC contents were measured in CD4 and CD8 T-cell populations of a small number of subjects in this original study. Reduced TREC contents in subjects with HIV-1 infection was confirmed in 2 additional studies $^{4,5}$ performed either on total blood mononuclear cells of subjects with chronic infection $(n=127)$ or on purified CD4 and CD8 T-cell populations of subjects with recent infection (12-18 months after acute infection; $\mathrm{n}=22$ ). However, rela- tively normal TREC contents were found in a study ${ }^{6}$ performed on total blood mononuclear cells of 74 subjects with established chronic infection. Hazenberg at $\mathrm{al}^{7}$ propose that the decline in TREC contents in HIV-1 infection results from increased cell division, not from thymus dysfunction. The latter study was performed in purified naive CD4 and CD8 T-cell populations from 33 subjects with advanced chronic infection.

Numerous studies suggest a relationship between the restoration of CD4 T-cell numbers after antiretroviral therapy (ART) and the production of thymic T cells..$^{3,8-16}$ Similarly, normal TREC contents have been observed after successful ART. ${ }^{17}$ However, because introducing only a few TREC-holding cells in a virtually empty CD4 T-cell pool may suffice to increase the average TREC content, it is debatable whether rapid normalization of CD4 TREC contents truly reflects increased thymic output. ${ }^{2,18}$

Because of the limited number of subjects analyzed in the above studies and, in particular, the small number of subjects studied for TREC contents in purified CD4 and CD8 T-cell populations, it has not been possible to address the impact of different stages of HIV-1 infection on TREC contents. In the present study, we hypothesized that thymic output may be affected differently, depending on patient age and on stage of HIV-1 infection. To test this hypothesis, TREC contents were measured in purified CD4 and CD8 T-cell
From the Laboratory of AIDS Immunopathogenesis, Division of Immunology and Allergy, Centre Hospitalier Universitaire Vaudois (CHUV), Lausanne, Switzerland; Laboratory of Molecular Immuno-Biology, Hospital General Universitario Gregorio Marañón (HGUGM), Madrid, Spain; Department of Clinical Viro-Immunology, Sanquin Research at Centraal Laboratorium van de Bloedtransfusiedienst van het Nederlandse Rode Kruis (CLB) and Landsteiner Laboratory, Academic Medical Center, University of Amsterdam, The Netherlands; Service Régional Vaudois de Transfusion Sanguine, Lausanne, Switzerland; and Theoretical Biology, Utrecht University, The Netherlands.

Submitted December 15, 2003; accepted March 11, 2004. Prepublished online as Blood First Edition Paper, April 1, 2004; DOI 10.1182/blood-2003-12-4265.

Supported by research grants from the Swiss National Foundation (FN 3100058913/2), the Dutch AIDS Foundation (7011), and the Netherlands
Organization for Scientific Research (916.36.003). In addition, this study has been financed in the framework of the Swiss HIV Cohort Study, supported by the Swiss National Science Foundation.

A complete list of the members of the Swiss HIV Cohort Study appears in the "Appendix."

Reprints: Giuseppe Pantaleo, Laboratory of AIDS Immunopathogenesis, Division of Immunology and Allergy, Centre Hospitalier Universitaire Vaudois, Rue Bugnon, 1011 Lausanne, Switzerland; e-mail: giuseppe.pantaleo@chuv.hospvd.ch.

The publication costs of this article were defrayed in part by page charge payment. Therefore, and solely to indicate this fact, this article is hereby marked "advertisement" in accordance with 18 U.S.C. section 1734.

(C) 2004 by The American Society of Hematology 
populations isolated from $297 \mathrm{HIV}$-1-infected subjects with established chronic infection and with no history of ART and in 120 HIV-negative subjects.

\section{Patients, materials, and methods}

\section{Study subjects and samples}

Blood samples of HIV-negative subjects $(\mathrm{n}=120)$ between 20 and 59 years of age (mean \pm SE, $37.9 \pm 0.99$ ) were randomly collected from the Service Régional Vaudois de Transfusion Sanguine of Lausanne.

Cryopreserved peripheral blood mononuclear cell (PBMC) samples from HIV-1-infected subjects between 20 and 60 years of age (37.01 \pm 0.58 years) with established HIV-1 infection (more than 4 years) were provided by the Swiss HIV Cohort $(n=297)$. Samples had been collected before the initiation of antiretroviral therapy. The CD4 T-cell count for the total cohort of patients with chronic established infection was $456 \pm 15.3$ cells $/ \mu \mathrm{L}$, and the viral load was $82907 \pm 11175$ copies/mL plasma. Viral loads for HIV-1-infected subjects were $35938 \pm 8677$ copies $/ \mathrm{mL}$ for patients with CD4 T-cell counts greater than 500; $97807 \pm 21801$ copies $/ \mathrm{mL}$ for patients with CD4 T-cell counts between 500 and 200 copies $/ \mathrm{mL}$; and $149221 \pm 27529$ for patients with CD4 T-cell counts less than 200 copies $/ \mathrm{mL}$. Biologic specimens from HIV-1-infected and HIV-negative subjects were collected under study protocols approved by the Institutional Review Board of the Centre Hospitalier Universitaire Vaudois, and all subjects gave informed consent in accordance with the Declaration of Helsinki.

\section{Isolation of CD4 and CD8 T cells}

PBMCs were isolated from ethylenediaminetetraacetic acid (EDTA) whole blood by Ficoll-Paque Plus density gradient centrifugation (Amersham Pharmacia Biotech, Duebendorf, Switzerland), washed twice in phosphatebuffered saline (PBS) containing $2 \%$ fetal bovine serum (FBS), and directly used for CD4 and CD8 T-cell isolation and flow cytometry. CD4 and CD8 T cells were isolated from total PBMCs of HIV-1-positive and -negative subjects using Dynal (Compiegne, France) $\mathrm{CD}^{+}{ }^{+}$and $\mathrm{CD} 8^{+}$isolation kits. Cells were isolated using an optimized protocol. Briefly, total PBMCs were resuspended in PBS containing 2\% FBS at a concentration of $5 \times 10^{6}$ cells/mL. CD4 or CD8 isolation beads previously washed twice in PBS containing $2 \%$ FBS were added at a ratio of 4 to 10 beads per positive cell and were incubated at $4{ }^{\circ} \mathrm{C}$ for 30 minutes under constant rotation. After incubation, beads were washed 5 times in PBS containing 2\% FBS on the Dynal magnet, resuspended in $100 \mu \mathrm{L}$ RPMI 1640 containing 1\% FBS supplemented with $10 \mu \mathrm{L}$ Detachbeads (Dynal), and incubated for 45 minutes at room temperature with constant shaking. Isolated cells were rescued in the supernatant using the Dynal magnet. Purity of the isolated CD4 and CD8 cells was always greater than $90 \%$. After counting, pellets of $0.25 \times 10^{6}$ cells were prepared to measure TREC contents.

\section{Measuring TRECs}

TRECs were determined using real-time polymerase chain reaction (PCR) in an ABI Prism 7700 thermocycler (Applied Biosystems, Cheshire, United Kingdom). DNA was extracted from CD4 or CD8 purified $\mathrm{T}$ cells by incubation with $100 \mu \mathrm{g} / \mathrm{mL}$ proteinase $\mathrm{K}$ (Roche Diagnostics, Mannheim, Germany) for 1 hour at $56^{\circ} \mathrm{C}$ and then for 15 minutes at $95^{\circ} \mathrm{C}$. Real-time PCR was performed in a volume of $25 \mu \mathrm{L}$ containing $3.5 \mathrm{mM} \mathrm{MgCl}_{2}, 200$ $\mu \mathrm{M}$ dNTPs, $0.5 \mu \mathrm{M}$ forward (CACATCCCTTTCAACCATGCT) and reverse (GCCAGCTGCAGGGTTTAGG) primers, fluorescence-labeled specific probe $(0.2 \mu \mathrm{M})$ (ACACCTCTGGTTTTTGTAAAGGTGCCCACT) (Megabases, Evanston, IL), $0.625 \mathrm{U}$ platinum Taq DNA polymerase (Invitrogen, Basel, Switzerland), and $5 \mu \mathrm{L}$ cell lysate (equivalent to 50000 cells). Amplification conditions were 1 cycle at $95^{\circ} \mathrm{C}$ for 5 minutes, followed by 40 cycles of amplification $\left(95^{\circ} \mathrm{C}\right.$ for 30 seconds, $60^{\circ} \mathrm{C}$ for 1 minute). A standard curve was made with serial dilutions of a plasmid that included a 375-bp fragment of the TRECs sequence.

\section{Flow cytometry}

The purity of CD4 and CD8 cell populations was tested by surface staining with the TriTEST anti-CD4 (fluorescein isothiocyanate [FITC]), anti-CD8 (phycoerythrin [PE]), and anti-CD3 (peridin chlorophyll protein [PerCP]) (Becton Dickinson, Franklin Lakes, NJ). Cells were incubated for 30 minutes with antibodies, washed, and fixed in CellFix (Becton Dickinson). For Ki67 intracellular analysis, cells were subjected to surface staining with anti-CD4 (PE), anti-CD45RA (CyChr), and anti-CD8 (allophycocyanin) (Becton Dickinson) for 30 minutes at $4{ }^{\circ} \mathrm{C}$, fixed with $1 \%$ formaldehyde, $2 \%$ glucose, and $5 \mu \mathrm{M}$ Na-Azide in PBS, and incubated for 15 minutes in the dark. After washing, fixed cells were permeabilized with $0.1 \%$ Saponin and were incubated with anti-Ki67 (FITC) (DAKO, Glostrup, Denmark) for 15 minutes in the dark. All samples were analyzed using FACScalibur cell analyzer and CellQuest Software (Becton Dickinson).

\section{Statistical analysis}

All statistical tests were interpreted at the 5\% significance level $(P<.05)$. Linear regression analysis was performed to determine the degree of dependence of different variables on patient age (Figure 1). For comparisons between healthy and HIV-1-infected subjects, the Levene test was used to assess homogeneity of the variances, and an independent-samples Student $t$ test was used when variances were homogeneous. The Welch test was used when variances were not homogeneous (Figures 2, 3A, and 4A; Table 1). Analysis of variance (ANOVA) was performed to compare variables among the 3 groups of HIV-1-infected subjects stratified by CD4 T-cell counts. Pearson correlation test was used to determine the correlation between variables.
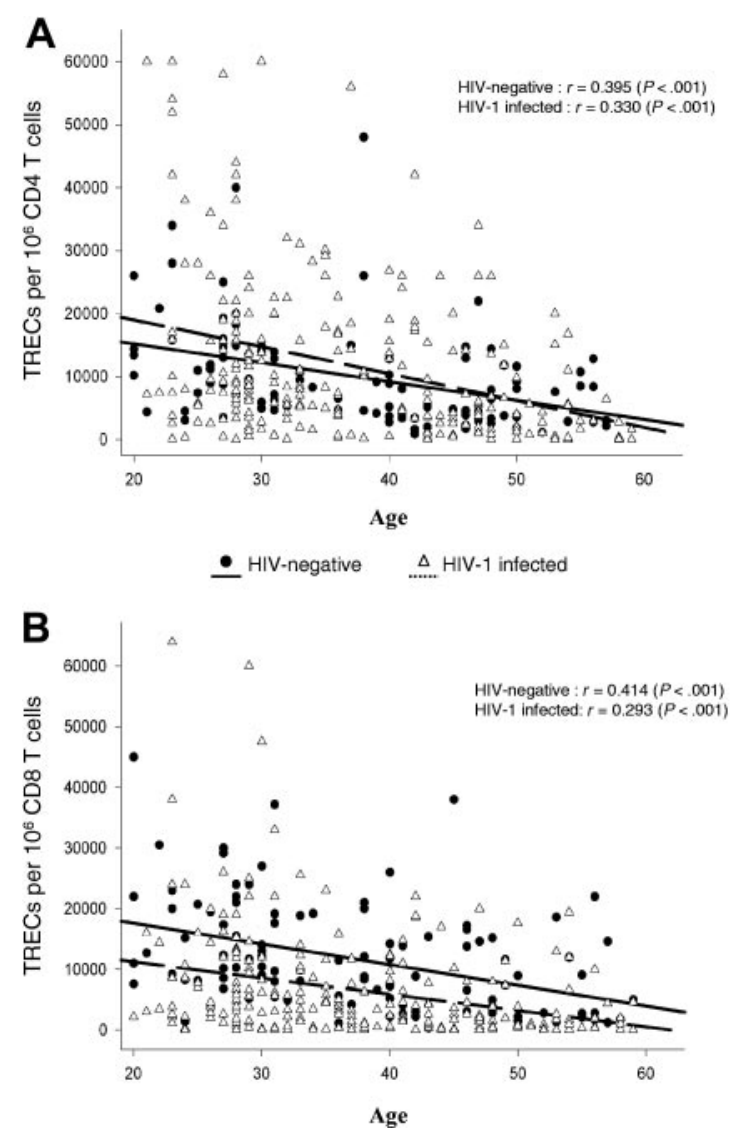

Figure 1. Correlation between TREC contents within CD4 and CD8 T-cell populations and age. Analysis was performed in $297 \mathrm{HIV}-1$-infected and 120 HIV-negative subjects ranging in age from 20 to 60 years. Continuous (HIV-negative) and dashed (HIV-1-infected) lines indicate best-fit linear regression curves, whereas r gives the Pearson correlation coefficient. TREC contents within purified CD4 (A) and CD8 (B) T-cell populations were determined as described in the "Patients, materials, and methods." 
A

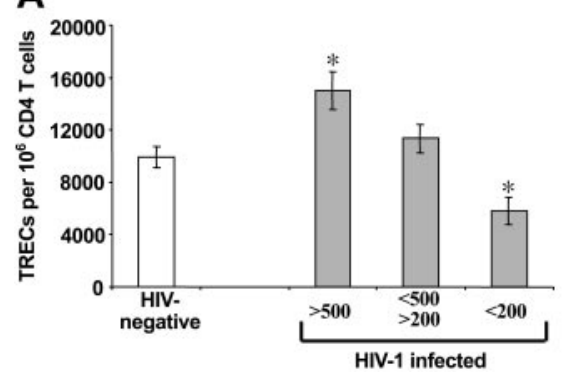

B

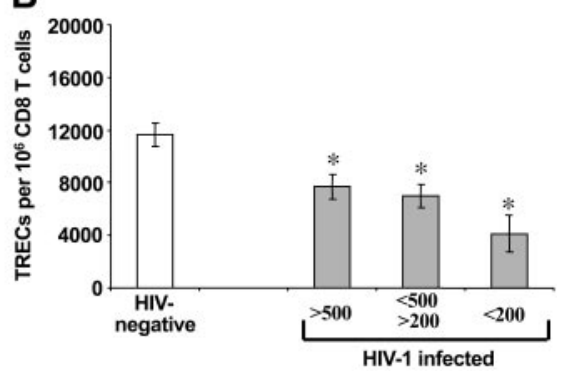

Figure 2. Comparative analysis of TREC contents within purified CD4 and CD8 T-cell populations of HIV-1-infected subjects at different stages of HIV-1 disease and HIV-negative subjects. Early-stage disease (more than $500 \mathrm{CD} 4 \mathrm{~T}$ cells), intermediate-stage disease (200-500 CD4 T cells), and late-stage disease (less than 200 CD4 T cells). Asterisks indicate statistical significance $(P<.05)$ between TREC contents within CD4 (A) and CD8 (B) T-cell populations in HIV-1-infected subjects (圆) and HIV-negative subjects ( $\square$ ). Data are expressed as mean $\pm \mathrm{SE}$.
To exclude that observed correlations between variables depended on age, the partial correlation test controlling for age was performed (Figures 3B and 4B). Multivariate regression analysis was used to study the effect and capacity of prediction of age, CD4 T-cell count, and viral load on TREC contents (Table 3).

\section{Results}

\section{Study groups}

HIV-1-infected subjects with chronic established infection and no previous history of ART and who had been enrolled in the Swiss HIV Cohort Study (SHCS) were randomly selected on the basis of CD4 T-cell counts. HIV-1-infected subjects in the present study had a mean time of documented diagnosis of approximately 4 years and had been infected for at least 1 year. Based on CD4 T-cell counts, the subjects were divided into 3 groups: CD4 T-cell counts greater than $500 / \mu \mathrm{L}$ (early-stage disease); CD4 T-cell counts 200-500/ $\mu \mathrm{L}$ (intermediate-stage disease), and CD4 T-cell counts lower than $200 / \mu \mathrm{L}$ (late-stage disease). The number of subjects studied included 117 subjects with early-stage disease, 123 with intermediate-stage disease, and 57 with late-stage disease. Overall, the 3 groups were well matched by sex (176 men, 121 women) and age (mean ages: 37.65 years for early stage, 36.91 years for intermediate stage, and 35.93 years for late stage). The slight differences in age among the different groups were not statistically significant $(P>.05)$. Most age groups (20-29, 30-39, and 40-49 years) contained a similar number of subjects (approximately $70-75)$, but the number was lower $(n=33)$ in the 50- to 59-yearold group. As a control group, 120 randomly selected, healthy, HIV-negative blood donors were recruited. The control group was matched by sex ( 60 males and 60 females) and by age ( $37.9 \pm 0.99$ years) compared with the HIV-1-infected subjects $(37.01 \pm 0.58$;
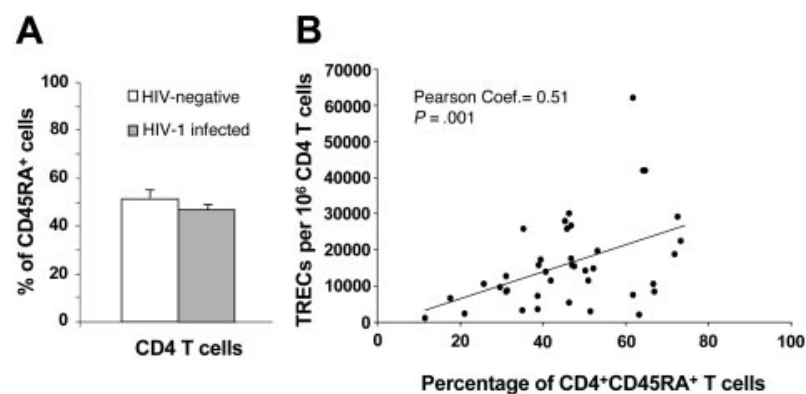

Figure 3. Naive CD4 T cells and TREC contents. (A) Comparative analysis between the percentage of CD45RA ${ }^{+}$CD4 ${ }^{+} T$ cells in HIV-negative subjects $(\square)$ and in 42 randomly selected HIV-1-infected subjects (圆) at early and intermediate stages of disease. (B) Correlation between the percentage of CD45RA $+C D 4+T$ cells and TREC contents within the CD4 T-cell population in HIV-1-infected subjects. Data are expressed as mean $\pm \mathrm{SE}$.
$P>.05)$. Furthermore, approximately 30 subjects were contained in each of the 3 age groups between 20 and 49 years, whereas the number of subjects was lower (approximately 15) in the 50- to 59-year-old group.

\section{Comparative analysis of TREC contents in HIV-1-infected subjects at different stages of disease and in healthy HIV-negative subjects}

Frozen blood mononuclear cell samples of the 297 HIV-1-infected subjects were obtained from the different laboratory repositories of the SHCS, and mononuclear cell samples of 120 HIV-negative subjects were prospectively collected from the Service Régional Vaudois de Transfusion Sanguine. CD4 and CD8 T-cell populations of HIV-1-infected and HIV-negative subjects were isolated from total mononuclear cells using CD4 and CD8 magnetic beads, as described in "Patients, materials, and methods." Previous studies have shown a negative correlation between age and TREC contents. The relationship between age and TREC contents of CD4 and CD8 T cells isolated from the 297 HIV-1-infected subjects and 120 healthy HIV-negative subjects is shown in Figure 1. Results confirmed a significant negative correlation (Figure 1).
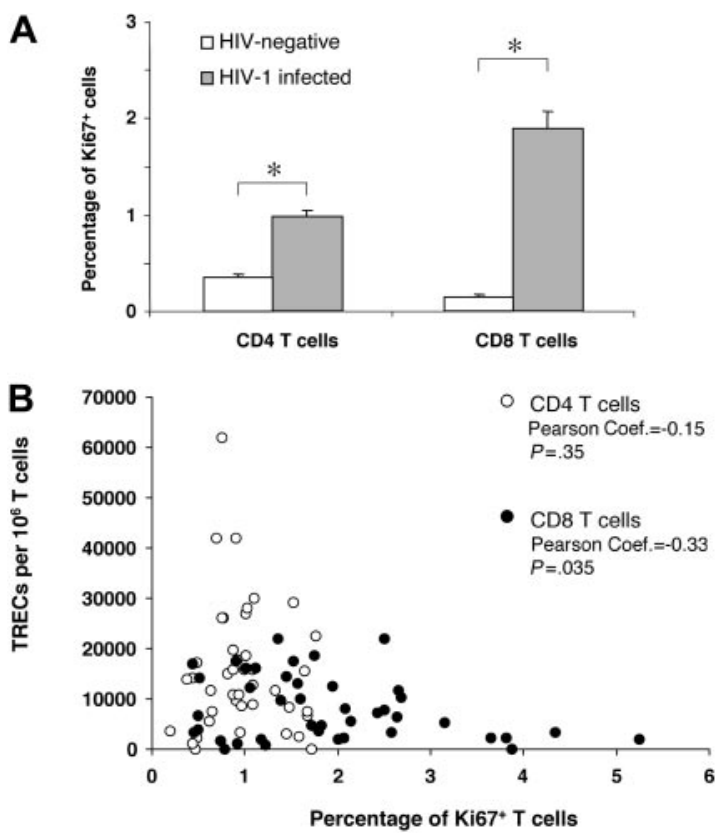

Figure 4. Impact of Ki67 expression on CD4 and CD8 TREC contents. (A) Comparative analysis between the percentage of Ki67+ CD4 and CD8 T cells in HIV-negative subjects ( $\square$ ) and in 42 randomly selected HIV-1-infected subjects ( $\square$ ) at early and intermediate stages of disease. (B) Correlation between the percentage of Ki67 ${ }^{+}$ CD4 $(\bigcirc)$ and CD8 $(\bullet)$ T cells and TREC contents within the 2 T-cell populations. Asterisks indicate statistical significance $(P<.05)$. Data are expressed as mean $\pm \mathrm{SE}$. 
Table 1. Influence of age on CD4 T-cell TREC contents in HIV-1-infected subjects at different stages of disease

\begin{tabular}{|c|c|c|c|c|c|}
\hline \multirow[b]{2}{*}{ T-cell count and age, $y^{*}$} & \multicolumn{2}{|r|}{ HIV-negative } & \multirow[b]{2}{*}{$P$} & \multicolumn{2}{|r|}{ HIV-1-infected } \\
\hline & $\mathbf{N}$ & TREC contents, mean \pm SE & & $\mathbf{N}$ & TREC contents, mean \pm SE \\
\hline \multicolumn{6}{|l|}{ More than $500 \mathrm{CD} 4 \mathrm{~T}$ cells } \\
\hline $20-39$ & 54 & $13206 \pm 1245$ & $.016 \dagger$ & 56 & $19418 \pm 2203$ \\
\hline $40-44$ & 14 & $5174 \pm 945$ & $.015 \dagger$ & 11 & $16119 \pm 3698$ \\
\hline $45-49$ & 18 & $7293 \pm 1319$ & .305 & 18 & $10063 \pm 2295$ \\
\hline $50-54$ & 9 & $5532 \pm 1223$ & .865 & 7 & $5133 \pm 1899$ \\
\hline $55-59$ & 7 & $6921 \pm 1608$ & $.029 \dagger$ & 7 & $2252 \pm 811$ \\
\hline \multicolumn{6}{|l|}{200 to $500 \mathrm{CD} 4 \mathrm{~T}$ cells } \\
\hline $20-39$ & 54 & $13206 \pm 1245$ & .863 & 65 & $13554 \pm 1506$ \\
\hline $40-44$ & 14 & $5174 \pm 945$ & .102 & 17 & $9089 \pm 2088$ \\
\hline $45-49$ & 18 & $7293 \pm 1319$ & .682 & 5 & $8616 \pm 3879$ \\
\hline $50-54$ & 9 & $5532 \pm 1223$ & .774 & 12 & $6235 \pm 1871$ \\
\hline $55-59$ & 7 & $6921 \pm 1608$ & $.014 \dagger$ & 3 & $1023 \pm 989$ \\
\hline \multicolumn{6}{|l|}{ Fewer than 200 CD4 T cells } \\
\hline $20-39$ & 54 & $13206 \pm 1245$ & $.005 \dagger$ & 30 & $7406 \pm 1463$ \\
\hline $20-29$ & 31 & $14696 \pm 1573$ & .060 & 15 & $9135 \pm 2583$ \\
\hline $30-39$ & 23 & $11199 \pm 1977$ & $.047 \dagger$ & 15 & $5676 \pm 1331$ \\
\hline
\end{tabular}

Values are based on TRECs per $10^{6}$ CD4 T cells. $P$ values represent $t$ test comparisons between values in the 2 groups.

*T-cell counts given are those of HIV-1-infected individuals in that group. Subgroups are stratified by age (in years).

†Statistically significant values.

We then compared the TREC contents in the CD4 and CD8 T-cell populations isolated from the 3 groups of HIV-1-infected subjects stratified by CD4 T-cell counts with those found in the same T-cell populations of healthy HIV-negative subjects. TREC numbers per $10^{6}$ CD4 $\mathrm{T}$ cells were $9952 \pm 801$ in healthy HIV-negative subjects $(\mathrm{n}=102)$ (Figure 2A). They were significantly higher $(P=.003)$ $15000 \pm 1484$ - in HIV-1-infected subjects $(\mathrm{n}=100)$ with CD4 T-cell counts greater than 500. TREC contents in CD4 T cells of HIV-1infected subjects with CD4 T-cell counts between 200 and 500 $(n=102)$ were also higher $(11338 \pm 1100)$ than in HIV-negative subjects, but the differences were not statistically significant $(P=.31)$ (Figure 2A). In contrast, TREC contents in CD4 T cells of HIV-1infected subjects with CD4 T-cell counts less than $200(\mathrm{n}=44)$ were $5807 \pm 1075$, significantly lower $(P=.004)$ than in HIV-negative subjects (Figure 2A).

Mean TREC contents per $10^{6}$ CD8 T cells were $7672 \pm 932$ in HIV-1-infected subjects with CD4 T-cell counts greater than 500 ( $\mathrm{n}=91), 7011 \pm 887$ with CD4 T-cell counts from 200 to 500 $(\mathrm{n}=99)$, and $4068 \pm 1373$ with CD4 T-cell counts less than 200 $(\mathrm{n}=47)$ (Figure 2B). Mean TREC contents found in CD8 T cells of the 3 groups of HIV-1-infected subjects were all significantly lower $(P<.003)$ than those of healthy HIV-negative subjects (11 $658 \pm 856 ; \mathrm{n}=109$ ) (Figure 2B). These results indicate a dichotomy between TREC contents in CD4 and CD8 T-cell populations in HIV-1 infection, and they illustrate the risk associated with interpreting changes in TREC contents of total PBMCs.

\section{Factors potentially influencing TREC contents}

We aimed to investigate why CD4 TREC contents in HIV-1infected subjects at early- and intermediate-stage disease were increased or were not decreased compared with healthy controls. Given that controls and subjects were age matched, these TREC differences could not be attributed to differences in age between the groups. Increased CD4 TREC contents could indicate more efficient thymic output in these groups of subjects compared with healthy controls. Previous studies have shown, however, that TREC contents also correlate with naive T cells, cell division, and cell death. ${ }^{3,5,7,15,18-22}$ It was, therefore, important to determine whether the increased TREC contents in HIV-1-infected subjects at early stages of disease were caused by differences in these factors.

\section{Naive CD4 T cells}

We investigated the fraction of naive CD4 T cells in 21 randomly selected subjects of the HIV-negative cohort and in 42 HIV-1-infected subjects at early- and intermediate-stage disease. The percentage of naive CD4 T cells, as measured by the expression of the CD45RA antigen, was not significantly different $(P=.3)$ in HIV-negative and HIV-1-infected subjects (Figure 3A). In line with previous studies, the percentage of naive CD4 T cells correlated positively with CD4 TREC contents in HIV-1-infected subjects (Figure 3B). Because we used frozen mononuclear cell populations, it was not possible to use CD62L to define naive T-cell subsets. However, previous studies have clearly shown that CD45RA defines at least 95\% of naive CD4 T cells. Therefore, though CD45RA is not a good marker of naive CD8 T cells, it is a valid marker of naive CD4 T cells. ${ }^{23,24}$

\section{Cell division}

Cell division was evaluated by expressing a nuclear antigen, Ki67, which is expressed in cycling cells, ${ }^{25,26}$ within the same group of 42 subjects at early- and intermediate-stage disease. It is important to underscore that recent studies have shown that the estimates of dividing CD4 and CD8 T cells, measured by Ki67 or by in vivo labeling studies with deuterated glucose, were comparable. ${ }^{27}$ As in previous studies, the percentages of $\mathrm{Ki}^{+} 7^{+}$in total CD4 and CD8 T cells were significantly higher $(P<.001)$ in HIV-1-infected subjects than in the HIV-negative control group (Figure 4A). ${ }^{28-30}$ We then analyzed the relationship between the extent of cell division and TREC contents. Percentages of $\mathrm{Ki}^{+}{ }^{+} \mathrm{CD} 4 \mathrm{~T}$ cells were so low that no correlation between the percentage of $\mathrm{Ki} 67^{+} \mathrm{CD} 4^{+} \mathrm{T}$ cells and CD4 TREC contents could be found (Figure $4 \mathrm{~B}$ ), whereas there was a clear negative correlation between the percentages of $\mathrm{Ki}^{+} 7^{+} \mathrm{CD} 8^{+} \mathrm{T}$ cells and $\mathrm{CD} 8 \mathrm{TREC}$ contents (Figure 4B). Summarizing, we could rule out the possibility that the increased CD4 TREC contents of patients with early- and intermediate-stage disease were caused by larger naive CD4 T-cell fractions or lower proliferation rates.

\section{CD4 T-cell death}

It is well known that CD4 T-cell death rates increase during HIV infection, and increased T-cell deaths increase CD4 TREC content by shortening the life expectancy of CD4 T cells. To 
determine whether the increased CD4 TREC contents of HIVinfected subjects at early stages of disease were merely the result of increased CD4 T-cell death, we calculated total CD4 TREC numbers per microliter blood in all groups of subjects and in controls. The advantage of measuring total TRECs instead of TREC contents is that total TREC numbers are only affected by thymic output and T-cell death; therefore, they reflect thymic output more directly than TREC contents, which are also affected by T-cell proliferation. TREC totals per microliter blood were calculated by multiplying the CD4 TREC contents with the CD4 T-cell counts per microliter blood for all subjects. Because exact CD4 counts in healthy controls were not available, we estimated these counts to be 1000 cells $/ \mu \mathrm{L}$. This is likely to be an overestimate given that the average CD4 T-cell count of HIV-negative subjects from the same blood bank, as determined in a previous study, ${ }^{25}$ was $856 \mathrm{CD} 4 \mathrm{~T}$ cells $/ \mu \mathrm{L}$ blood. Even using this overestimate, we found that patients with high CD4 T-cell counts (and increased CD4 TREC contents), had larger total numbers of CD4 TRECs per microliter blood (11 100) than healthy controls. If the observed increased (Figure 2) CD4 TREC contents in these patients were attributed to HIV-induced T-cell death, we would have expected to find decreased total CD4 TREC numbers instead, because T-cell death can only lead to a reduction of total TRECs. Apparently, the production of new TREC-holding T cells in early-stage HIV infection sufficed to compensate for the loss of TRECs by increased T-cell death. Having ruled out all other possible mechanisms for the increased TREC contents in early-stage disease, we concluded that thymic output was indeed increased in HIV-1-infected subjects compared with healthy controls.

\section{Influence of age on CD4 T-cell TREC contents in HIV-1-infected subjects at different stages of HIV-1 disease}

To assess the influence of age on CD4 TREC contents, de novo T-cell generation was compared between HIV-1-infected subjects and HIV-negative subjects at different stages of disease, stratified by age. We observed that CD4 TREC contents were significantly higher (Table 1) in HIV-1-infected subjects with CD4 T-cell counts greater than 500 who were between 20 and 44 years of age, whereas no significant increases in CD4 TREC contents were found in subjects between 45 and 59 years of age (Table 1). To better characterize the influence of age on CD4 TREC contents, we further stratified those aged 40 to 59 in 5-year subgroups (40-44, 45-49, 50-54, and 55-59 years). CD4
TREC contents were significantly higher for patients between 20 and 39 years, and they were still higher in HIV-1-infected subjects between 40 and 44 years (Table 1). No significant differences between HIV-1-infected and HIV-negative subjects were observed in the 45- to 49-year-old and the 50- to 54-year-old groups (Table 1). CD4 TREC contents were even significantly lower in HIV-1-infected subjects than in HIVnegative subjects in the 55- to 59-year-old group (Table 1). In HIV-1-infected subjects between 20 and 54 with CD4 T-cell counts between 200 and 500, CD4 TREC contents were not significantly different from those of healthy controls, but they were significantly lower in 55- to 59-year-old subjects (Table 1). In 20- to 39-year-old HIV-1-infected subjects with CD4 T-cell counts lower than 200, CD4 TREC contents were significantly lower than they were in healthy controls, and further analysis showed that they were substantially lower in subjects 20 to 29 years of age (Table 1).

These results indicate that the increase in de novo CD4 T-cell generation is mostly limited to younger (younger than 45 years) HIV-1-infected subjects during early-stage disease. To address the impact of age on CD4 TREC contents in more detail, we determined the proportion of patients with CD4 TREC contents greater than, similar to, or lower than the mean $\pm 3 \times \mathrm{SE} \mathrm{CD} 4$ TREC contents in age-matched HIV-negative subjects (Table 2). Subjects were divided into 2 age groups, 20 to 44 and 45 to 59 . Dividing subjects in this way was based on the observation that the 40- to 50-year age range seems to define a critical period in thymus function. The highest proportion $(51 \%)$ of HIV-1-infected subjects with CD4 TREC contents higher than those in age-matched HIV-negative subjects was found in the younger age group (20-44 years) with CD4 T-cell counts greater than 500 (Figure 5). Approximately $20 \%$ had similar CD4 TREC contents and $28 \%$ had lower CD4 TREC contents than HIV-negative subjects (Figure 5). The proportion of HIV-1-infected subjects with CD4 TREC contents lower than those of healthy controls was substantially increased $(50 \%)$ in the 45- to 59-year-old group (Figure 5). In patients with CD4 T-cell counts between 200 and 500, approximately $30 \%$ of each age group had increased CD4 TREC contents, whereas approximately 50\% had lower CD4 TREC contents than healthy age-matched controls (Figure 5). Finally, approximately $80 \%$ of 20 - to 44 -year-old subjects with CD4 T-cell counts lower than 200 had CD4 TREC contents lower than those of age-matched HIV-negative subjects (Figure 5). These analyses further support the observation that de novo CD4 T-cell generation is selectively

Table 2. CD4 TREC contents in 3 subgroups of HIV-1-infected subjects identified by number of CD4 TRECs compared with HIV-negative subjects

\begin{tabular}{|c|c|c|c|c|c|c|c|c|c|c|c|}
\hline \multirow{2}{*}{$\begin{array}{c}\text { Age and } \\
\text { HIV-1-infected } \\
\text { subject } \\
\text { group* }^{*}\end{array}$} & \multicolumn{2}{|c|}{ HIV-negative subjects } & \multicolumn{3}{|c|}{ More than $500 \mathrm{CD} 4 \mathrm{~T}$ cells } & \multicolumn{3}{|c|}{200 to $500 \mathrm{CD} 4 \mathrm{~T}$ cells } & \multicolumn{3}{|c|}{ Fewer than $200 \mathrm{CD} 4 \mathrm{~T}$ cells } \\
\hline & $\mathbf{N}$ & $\begin{array}{c}\text { CD4 TRECs, } \\
\text { mean } \pm 3 \times \text { SE }\end{array}$ & $\mathbf{N}$ & CD4 TRECs & $P$ & $\mathbf{N}$ & CD4 TRECs & $\boldsymbol{P}$ & $\mathbf{N}$ & CD4 TRECs & $P$ \\
\hline $20-44$ y & 68 & $11553 \pm 3240$ & & & & & & & & & \\
\hline Higher & & & 34 & $30615 \pm 2394$ & $<.001 \dagger$ & 27 & $25541 \pm 2204$ & $<.001 \dagger$ & 3 & $24487 \pm 8757$ & $.019 \dagger$ \\
\hline Similar to & & & 14 & $11055 \pm 525$ & .680 & 18 & $10549 \pm 474$ & .397 & 5 & $11790 \pm 867$ & .953 \\
\hline Lower & & & 19 & $3634 \pm 599$ & $<.001 \dagger$ & 37 & $4216 \pm 398$ & $<.001 \dagger$ & 26 & $3802 \pm 581$ & $<.001 \dagger$ \\
\hline $45-59$ y & 34 & $6750 \pm 2481$ & & & & & & & & & \\
\hline Higher & & & 8 & $19550 \pm 2849$ & $.002 \dagger$ & 6 & $15143 \pm 1760$ & $<.001 \dagger$ & NA & NA & NA \\
\hline Similar to & & & 8 & $5760 \pm 236$ & .257 & 4 & $5550 \pm 492$ & .224 & NA & NA & NA \\
\hline Lower & & & 16 & $1896 \pm 351$ & $<.001 \dagger$ & 10 & $791 \pm 308$ & $<.001 \dagger$ & NA & NA & NA \\
\hline
\end{tabular}

$P$ values represent $t$ test comparisons between values in both groups. NA indicates not applicable.

*HIV-1-infected subjects are divided by age (in years) and subdivided according to whether their TREC counts were higher, similar to, or lower than those of their HIVnegative counterparts.

†Statistically significant values. 


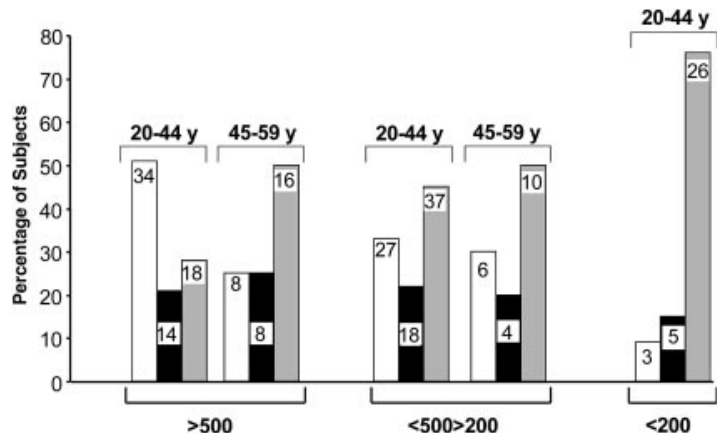

Figure 5. Proportion of HIV-1-infected subjects with CD4 TREC contents greater than, similar to, and lower than those in HIV-negative subjects. Groups with greater or lower TREC contents were defined by CD4 TREC contents greater or lower than the mean $\pm 3 \times$ SE CD4 TREC contents in age-matched HIV-negative subjects. Proportions were calculated in patients within 2 age groups (20-44 and 45-59 years) at different stages of disease. Numerical values within the bars indicate the number of subjects analyzed per group. Higher TREC contents ( $\square$ ). Similar TREC contents ( $\square)$. Lower TREC contents (䲆).

increased in younger HIV-1-infected patients and that this increase is predominantly found in patients during early-stage disease.

\section{CD4 T-cell counts and TREC contents}

The data shown in Figure 2A indicate that CD4 TREC contents were similar between HIV-1-infected subjects and HIV-negative subjects at intermediate-stage disease and that they became significantly lower in subjects with advanced-stage disease. To better define the CD4 T-cell count corresponding to the significant reduction of CD4 TREC contents, HIV-1-infected subjects at intermediate- and late-stage disease were stratified further by smaller ranges of CD4 T-cell counts: 400 to 200, 300 to 200, and 200 to 100 . The mean CD4 T-cell count was $315.7 \pm 51.9$ in the HIV-1-infected subjects $(n=65)$ with 400 to 200 CD4 T cells. In this group, the mean CD4 TREC content was $11653 \pm 1467$, not significantly different $(9952 \pm 801 ; P=.3)$ from that of HIVnegative subjects $(\mathrm{n}=102)$ (Figure 6$)$. The mean CD4 T-cell count was $257.6 \pm 29.3$ in the HIV-1-infected subjects $(\mathrm{n}=21)$ with CD4 T cells between 300 and 200, and the mean CD4 TREC content $(9217 \pm 1763)$ was not significantly different $(P=.7)$ from that of HIV-negative subjects (Figure 6). The mean CD4 T-cell count was $153 \pm 25$ in the HIV-1-infected subjects $(n=28)$ with CD4 T-cell counts between 200 and 100, and the mean CD4 TREC content $(6412 \pm 1613)$ was significantly lower $(P=.045)$ than in HIV-negative subjects (Figure 6).

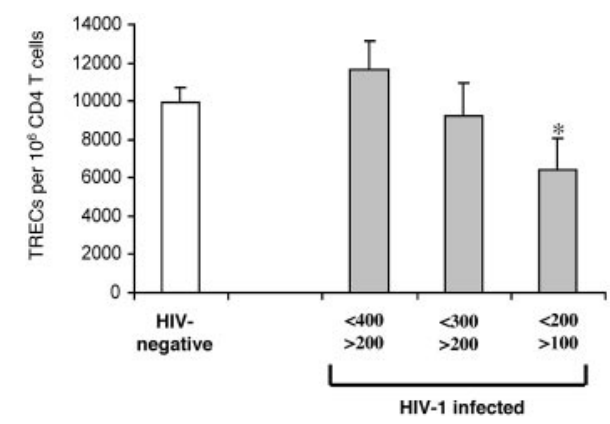

Figure 6. Identifying CD4 T-cell counts associated with reduced TREC contents within CD4 T cells. CD4 TREC contents were compared in 3 groups of HIV-1infected subjects (圆) with CD4 T-cell counts from 400 to 200,300 to 200 , and 200 to 100 cells and of HIV-negative subjects $(\square)$. Asterisk indicates statistical significance $(P<.05)$. Data expressed as mean \pm SE.

\section{Viral load and TREC contents}

Analysis of the entire cohort revealed a negative correlation between viral load and CD4 TREC contents $(P=.015)$. However, when patients were stratified by viral load (eg, fewer than 25000 , between 25000 and 100000 , and more than 100000 ), significant differences in CD4 TRECs in HIV-1-infected and HIV-negative subjects were found only in those with a viral load less than 25000 HIV-1 RNA copies per milliliter plasma. These subjects appeared to have significantly higher CD4 TREC contents than healthy controls $(P=.001)$. Approximately $60 \%$ of the subjects in this group had CD4 T-cell counts higher than 500 per microliter blood. Therefore, low viral load seems to be important for increased T-cell generation (data not shown).

\section{Factors predicting TREC contents}

On the basis of our results, age, CD4 T-cell count, and viral load may influence CD4 TREC contents. We evaluated the ability of these factors to serve as predictors of CD4 TREC contents by multivariate regression analysis with age, CD4 T-cell counts, and viral load as predictors (constant) and TREC contents as the dependent variable. This analysis was performed on the total cohort of 297 subjects enrolled in the study (Table 3). Age was the strongest predictor of TREC contents in CD4 T cells. CD4 T-cell count was also a good predictor of CD4 TREC contents. In contrast, viral load was not a predictor of TREC contents in CD4 T cells. Similarly, age and CD4 T-cell count, but not viral load, were good predictors of TREC contents in CD8 T cells (data not shown).

\section{Discussion}

The purpose of the present study was to determine the impact of age and the stages of HIV-1 infection on de novo T-cell generation by determining TRECs. Several studies that have recently investigated the effects of HIV-1 infection on TRECs concluded that HIV-1 infection severely affects thymus function. ${ }^{3-6}$ In general, however, the number of subjects studied was relatively small, particularly in studies in which TREC contents were determined in purified CD4 and CD8 T cells. Therefore, they were not statistically powered to assess the impact of the different stages of HIV-1 disease and of factors such as age on TREC contents in CD4 and CD8 T cells. For these reasons, we undertook an extensive analysis of TREC contents and total TRECs in purified CD4 and CD8 T cells in 2 large cohorts of HIV-1-infected $(\mathrm{n}=297)$ and of HIV-negative subjects $(n=120)$. HIV-1-infected subjects were stratified by CD4 T-cell counts corresponding to early, intermediate, and late stages of HIV-1 disease. The impact of age was also determined.

Table 3. Multiregression analysis of the predictive ability of age, CD4 T-cell count, and viral load on TREC contents within CD4 T cells

\begin{tabular}{lrcc}
\hline Variable & Coefficient & 95\% Cl & \multicolumn{1}{c}{} \\
\hline Constant & 25573.52 & 16795.02 to 30352.02 & $<.001$ \\
Age & -431.45 & -592.52 to -270.38 & $<.001^{*}$ \\
CD4 count & 10.60 & 4.32 to 16.88 & $.001^{*}$ \\
Viral load & -0.007 & -0.017 to 0.002 & .138 \\
\hline
\end{tabular}

$\mathrm{Cl}$ indicates confidence interval.

*Statistically significant values. 
Our results indicated that CD4 and CD8 TREC contents are differently affected throughout the stages of HIV-1 infection. According to previous studies, TREC contents in CD8 T cells were severely reduced in HIV-1-infected subjects. ${ }^{3,7}$ We showed that they were reduced at any stage of HIV-1 disease. We found that the percentage of dividing CD8 T cells was significantly higher in HIV-1-infected subjects than in HIV-negative subjects and that dividing cells correlated negatively with TREC contents in CD8 T cells. These results supported the hypothesis of Hazenberg et $\mathrm{al}^{7}$ that a dilution effect caused by increased cell division may contribute greatly to the reduction of TREC contents in HIV-1 infection. ${ }^{28-30}$

TREC contents in CD4 $\mathrm{T}$ cells, however, were influenced differently throughout the stages of HIV-1 disease. Compared with HIV-negative subjects, TREC contents in CD4 T cells were significantly higher in HIV-1-infected subjects during early-stage disease, similar (or slightly increased) during intermediate-stage disease, and severely reduced only at late-stage disease. We excluded the possibility that some factors known to influence TREC contents, including age, percentage of naive $\mathrm{T}$ cells, and extent of cell division and of cell death, could explain the increased TREC contents in CD4 T cells of HIV-1-infected subjects at earlyand intermediate-stage disease. ${ }^{3,5,7,15,18-22}$ A larger fraction of naive $\mathrm{T}$ cells is, in principle, associated with higher TREC contents. We found similar percentages of naive CD4 T cells in HIV-1-infected and HIV-negative subjects. Furthermore, increased cell division may dilute TRECs, possibly reducing average TREC contents despite thymic function in the normal range. Consistent with previous studies, ${ }^{28-32}$ the percentage of dividing CD4 T cells was increased (approximately 2-fold) in HIV-1-infected subjects at early- and intermediate-stage disease compared with HIV-negative subjects. Therefore, the effect, if any, of increased percentages of dividing CD4 T cells would be reduced rather than increased CD4 TREC contents. By analyzing total TREC numbers per microliter blood, we also excluded the possibility that the increased CD4 TREC contents in early-stage HIV-1 infection were merely attributed to increased CD4 T-cell death rates. We conclude that de novo T-cell generation in HIV-1-infected subjects during early-stage disease was higher than in healthy age-matched controls. A question, however, arises. Why did we find evidence for increased CD4 TREC contents though previous studies do not report this and many even suggest impaired thymic function during HIV infection? As mentioned, the present study has a 2-fold advantage- the large number of subjects studied and the analysis of TRECs in purified CD4 and CD8 $\mathrm{T}$ cells. This strategy allowed us to investigate extensively the role of age, a factor known to influence thymus function, on de novo CD4 T-cell generation and thus on T-cell TREC contents. We found that increased de novo CD4 T-cell generation was primarily limited to HIV-1-infected subjects younger than 45 years during early-stage disease. We also quantified the proportion of subjects with increased de novo CD4 T-cell generation at different stages of HIV-1 disease; this analysis confirmed that the highest percentage of subjects with increased CD4 TREC contents was found during early-stage disease in those who were younger. Thus, the increase in CD4 TRECs compared with healthy controls was strictly age dependent and was attributed to a subgroup of patients (younger than 45 years) who, because of their small number, might have been underrepresented in previous studies. The increase was already lost in subjects in the 45- to 49-year range, and CD4 TREC contents became severely lower in subjects in the 55- to 59-year range. Increased de novo CD4 T-cell generation in younger patients during early-stage disease could represent a compensatory mechanism for the depletion of CD4 T cells or perhaps a response to high levels of cytokines expressed during HIV infection. ${ }^{33}$ Alternatively, the apparent increase in CD4 TREC contents in young HIV patients with relatively high CD4 counts might have resulted from high preseroconversion CD4 TREC contents in this group of patients. In either case, the current analysis underscores the central role of de novo T-cell generation in early HIV infection because it can either increase on HIV infection or remain large despite HIV infection.

Our results are in accordance with a series of previous reports suggesting the possibility of increased thymus function in HIV infection. A high prevalence of thymic tissue, as measured on chest tomography, was reported in a group of HIV-1-infected patients compared with healthy controls, and abundant thymic tissue was shown in patients younger than 40 years at early-stage disease. ${ }^{34}$ No decreased CD4 TREC contents have been found in HIV-1-infected adolescents compared with HIV-negative adolescents. ${ }^{35}$ Furthermore, studies in simian immunodeficiency virus (SIV)-infected monkeys with no signs of AIDS have shown increased numbers of mature thymocytes and increased cell proliferation in the thymus. ${ }^{36}$ Thymocyte numbers were severely reduced only in SIV-infected monkeys with clinical signs of AIDS.

It is, however, important to underscore that the increase of de novo T-cell generation could not be observed during later stages of disease. Even among young patients, only 10\% had increased CD4 TREC contents at advanced-stage disease. Consistent with this finding, isotopic labeling studies have shown that in HIV-1infected patients at advanced stages of disease (eg, CD4 T-cell counts less than 200), T-cell production did not increase despite a significantly increased T-cell turnover rate. ${ }^{31,37}$ The close relationship between age, stage of HIV infection, and increased de novo T-cell generation sheds light on some clinical issues associated with HIV-1 disease. Based on the present results and previous studies, the more rapid progression of HIV-1 disease in the elderly is likely the result of reduced or lost ability to compensate CD4 T-cell depletion with increased de novo T-cell generation. ${ }^{38,39}$ Similarly, T-cell reconstitution in patients with CD4 T-cell counts less than 200 who begin antiretroviral therapy may be hampered. ${ }^{40}$ The importance of CD4 T-cell counts, not of viral load, in influencing the rates of disease progression is also consistent with our finding of no substantial effects of viral load on TREC contents. ${ }^{40}$

The present study allows a few more considerations. If de novo T-cell generation of CD4 T cells is preserved during HIV infection, there is no reason CD8 T-cell generation cannot be preserved. Yet CD8 TREC contents were not increased in younger patients during early-stage HIV infection. This is because of the TREC-diluting effect of increased proliferation of CD8 T cells during HIV infection. Such a diluting effect must also have occurred for the CD4 T cells, but to a lesser extent so that it would not mask the increased de novo T-cell generation. Our study may also facilitate an understanding of why most of the studies performed on nonfractionated blood mononuclear cells suggest defective thymus function. ${ }^{4,5}$ By analyzing TREC contents in total blood mononuclear cells, it is highly likely that the severe reduction in CD8 TREC contents masked the enrichment of TRECs in CD4 T cells of younger patients during early-stage disease.

In conclusion, though previous investigators conclude that CD4 and CD8 TREC contents are decreased in HIV-1 infection, here we demonstrate that CD4 TREC contents do not decrease until 
late-stage disease. Our results indicate that de novo CD4 T-cell generation is preserved for a long time during the course of HIV infection and that it is even increased in young HIV-1-infected patients during early-stage disease. At more advanced stages of disease and in older patients, no such increased T-cell production could be observed.

\section{Acknowledgments}

We thank Kim Ellefsen for technical advice and Frank Miedema for helpful discussion.

\section{Appendix}

The members of the Swiss HIV Cohort Study are: S. Bachmann, M. Battegay, E. Bernasconi, H. Bucher, P. Bürgisser, S. Cattacin, M. Egger, P. Erb, W. Fierz, M. Fischer, M. Flepp, A. Fontana, P. Francioli (President of the SHCS, Centre Hospitalier Universitaire Vaudois, CH-1011-Lausanne), H.J. Furrer (Chairman of the Clinical and Laboratory Committee), M. Gorgievski, H. Günthard, B. Hirschel, L. Kaiser, C. Kind, T. Klimkait, U. Lauper, B. Ledergerber, M. Opravil, F. Paccaud, G. Pantaleo, L. Perrin, J.-C. Piffaretti, M. Rickenbach (Head of Data Center), C. Rudin (Chairman of the Mother and Child Substudy), J. Schüpbach, R. Speck, A. Telenti, A. Trkola, P. Vernazza (Chairman of the Scientific Board), R. Weber, and S. Yerly.

\section{References}

1. Kong F, Chen $\mathrm{CH}$, Cooper MD. Thymic function can be accurately monitored by the level of recent T cell emigrants in the circulation. Immunity. 1998;8:97-104.

2. Hazenberg MD, Borghans JA, de Boer RJ, Miedema F. Thymic output: a bad TREC record. Nat Immunol. 2003;4:97-99.

3. Douek DC, McFarland RD, Keiser PH, et al. Changes in thymic function with age and during the treatment of HIV infection. Nature. 1998;396:690-694.

4. Hatzakis A, Touloumi G, Karanicolas R, et al. Effect of recent thymic emigrants on progression of HIV-1 disease. Lancet. 2000;355:599-604

5. Douek DC, Betts MR, Hill BJ, et al. Evidence for increased T cell turnover and decreased thymic output in HIV infection. J Immunol. 2001;167: 6663-6668.

6. Zhang L, Lewin SR, Markowitz M, et al. Measuring recent thymic emigrants in blood of normal and HIV-1-infected individuals before and after effective therapy. J Exp Med. 1999;190:725-732.

7. Hazenberg MD, Ottom SA, Cohen Stuart JW, et al. Increased cell division but not thymic dysfunction rapidly affects the $T$-cell receptor excision circle content of the naive $T$ cell population in HIV-1 infection. Nat Med. 2000;6:1036-1042.

8. Natarajan V, Lempicki RA, Sereti I, et al. Increased peripheral expansion of naive $\mathrm{CD} 4^{+} \mathrm{T}$ cells in vivo after IL-2 treatment of patients with HIV infection. Proc Natl Acad Sci U S A. 2002;99: 10712-10717.

9. Napolitano LA, Lo JC, Gotway MB, et al. Increased thymic mass and circulating naive CD4 T cells in HIV-1-infected adults treated with growth hormone. AIDS. 2002;16:1103-1111.

10. Delgado J, Leal M, Ruiz-Mateos E, et al. Evidence of thymic function in heavily antiretroviraltreated human immunodeficiency virus type 1-infected adults with long-term virologic treatment failure. J Infect Dis. 2002;186:410-414.

11. De Rossi A, Walker AS, Klein N, De Forni D, King D, Gibb DM. Increased thymic output after initiation of antiretroviral therapy in human immunodeficiency virus type 1-infected children in the Paediatric European Network for Treatment of AIDS (PENTA) 5 Trial. J Infect Dis. 2002;186:312-320.

12. Kolte L, Dreves AM, Ersboll AK, et al. Association between larger thymic size and higher thymic output in human immunodeficiency virus-infected patients receiving highly active antiretroviral therapy. J Infect Dis. 2002;185:1578-1585.

13. Sempowski GD, Haynes BF. Immune reconstitution in patients with HIV infection. Annu Rev Med. 2002;53:269-284.

14. Teixeira L, Valdez H, McCune JM, et al. Poor CD4 $T$ cell restoration after suppression of HIV-1 repli- cation may reflect lower thymic function. AIDS. 2001;15:1749-1756.

15. Haynes BF, Markert ML, Sempowski GD, Pate DD, Hale LP. The role of the thymus in immune reconstitution in aging, bone marrow transplantation, and HIV-1 infection. Annu Rev Immunol. 2000;18:529-560.

16. Douek DC, Koup RA, McFarland RD, Sullivan JL, Luzuriaga K. Effect of HIV on thymic function before and after antiretroviral therapy in children. $J$ Infect Dis. 2000;181:1479-1482.

17. Steffens CM, Smith KY, Landay A, et al. T cell re ceptor excision circle (TREC) content following maximum HIV suppression is equivalent in HIVinfected and HIV-uninfected individuals. AIDS. 2001;15:1757-1764

18. Hazenberg MD, Otto SA, de Pauw ES, et al. T-cell receptor excision circle and T-cell dynamics after allogeneic stem cell transplantation are related to clinical events. Blood. 2002:99:3449-3453.

19. Clark DR, de Boer RJ, Wolthers KC, Miedema F. $T$ cell dynamics in HIV-1 infection. Adv Immunol. 1999;73:301-327.

20. Sprent J, Tough DF. Lymphocyte life-span and memory. Science. 1994;265:1395-1400.

21. Hazenberg MD, Stuart JW, Otto SA, et al. T-cell division in human immunodeficiency virus (HIV)-1 infection is mainly due to immune activation: a longitudinal analysis in patients before and during highly active antiretroviral therapy (HAART). Blood. 2000;95:249-255.

22. Unutmaz D, Pileri $\mathrm{P}$, Abrignani $\mathrm{S}$. Antigen-independent activation of naive and memory resting $T$ cells by a cytokine combination. J Exp Med. 1994:180:1159-1164.

23. De Rosa SC, Herzenberg LA, Herzenberg LA, Roederer M. 11-Color, 13-parameter flow cytometry: identification of human naive $T$ cells by phenotype, function, and T-cell receptor diversity. Nat Med. 2001;7:245-248.

24. Sallusto F, Lenig D, Forster R, Lipp M, Lanzavec chia A. Two subsets of memory T lymphocytes with distinct homing potentials and effector functions. Nature. 1999;401:708-712.

25. Gerdes J, Lemke $\mathrm{H}$, Baisch $\mathrm{H}$, Wacker $\mathrm{HH}$, Schwab U, Stein H. Cell cycle analysis of a cell proliferation-associated human nuclear antigen defined by the monoclonal antibody Ki-67. J Immunol. 1984;133:1710-1715.

26. Schwarting R, Gerdes J, Niehus J, Jaeschke L, Stein $\mathrm{H}$. Determination of the growth fraction in cell suspensions by flow cytometry using the monoclonal antibody Ki-67. J Immunol Methods. 1986:90:65-70.

27. Deeks SG, Hoh R, Grant RM, et al. CD4+ T cell kinetics and activation in human immunodeficiency virus-infected patients who remain viremic despite long-term treatment with protease inhibitor-based therapy. J Infect Dis. 2002;185:315-323.

28. Fleury S, Rizzardi, GP, Chapuis, A, et al. Long-term kinetics of T cell production in HIV-infected subjects treated with highly active antiretroviral therapy. Proc Natl Acad Sci U S A. 2000;97:5393-5397.

29. Fleury S, de Boer RJ, Rizzardi GP, et al. Limited CD4+ T-cell renewal in early HIV-1 infection: effect of highly active antiretroviral therapy. Nat Med. 1998;4:794-801.

30. Sachsenberg N, Perelson AS, Yerly S, et al. Turnover of CD4+ and CD8 + T lymphocytes in HIV-1 infection as measured by Ki-67 antigen. J Exp Med. 1998;187:1295-1303.

31. McCune JM, Hanley MB, Cesar D, et al. Factors influencing T-cell turnover in HIV-1-seropositive patients. J Clin Invest. 2000;105:R1-R8.

32. Hellerstein MK, McCune JM. T cell turnover in HIV-1 disease. Immunity. 1997;7:583-589.

33. Napolitano LA, Grant RM, Deeks SG, et al. Increased production of IL-7 accompanies HIV-1mediated T-cell depletion: implications for T-cell homeostasis. Nat Med. 2001;7:73-79.

34. McCune JM, Loftus R, Schmidt DK, et al. High prevalence of thymic tissue in adults with human immunodeficiency virus-1 infection. J Clin Invest. 1998;101:2301-2308.

35. Pahwa S, Chitnis V, Mitchell RM, et al. CD4+ and $\mathrm{CD} 8+\mathrm{T}$ cell receptor repertoire perturbations with normal levels of $\mathrm{T}$ cell receptor excision circles in HIV-infected, therapy-naive adolescents. AIDS Res Hum Retroviruses. 2003;19:487-495.

36. Sopper S, Nierwetberg D, Halbach A, et al. Impact of simian immunodeficiency virus (SIV) infection on lymphocyte numbers and T-cell turnover in different organs of rhesus monkeys. Blood. 2003;101:1213-1219.

37. Hellerstein M, Hanley MB, Cesar D, et al. Directly measured kinetics of circulating $T$ lymphocytes in normal and HIV-1-infected humans. Nat Med. 1999;5:83-89.

38. Kalayjian RC, Landay A, Pollard RB, et al. Agerelated immune dysfunction in health and in human immunodeficiency virus (HIV) disease: association of age and HIV infection with naive CD8+ cell depletion, reduced expression of CD28 on CD8+ cells, and reduced thymic volumes. J Infect Dis. 2003;187:1924-1933.

39. Babiker AG, Peto T, Porter K, Walker AS, Darbyshire $\mathrm{JH}$. Age as a determinant of survival in HIV infection. J Clin Epidemiol. 2001;54(suppl 1):S16-S21.

40. Hogg RS, Yip B, Chan KJ, et al. Rates of disease progression by baseline CD4 cell count and viral load after initiating triple-drug therapy. JAMA. 2001;286:2568-2577. 